\title{
Response of a double system beam and string with an elastic layer to the dynamic excitations
}

\author{
L. Frýba, C. Fischer \& Sh. Urushadze \\ Institute of Theoretical and Applied Mechanics, \\ Academy of Sciences of the Czech Republic, Prague
}

\begin{abstract}
The beam with an axial force is coupled with the pretensiled string by an elastic layer of Winkler type. It is subjected to a row of moving forces. The theoretical model corresponds to a prestressed bridge. The governing equations form a coupled set of partial differential equations which are solved using the Fourier and Laplace-Carson integral transformation methods. A simple experimental model was constructed in a form of a plexiglass beam and two strings bound together with several spring and damping elements. The beam was excited by an electro-magnetic exciter and its response was measured at several places. Three steps of stiffness and damping characteristics of the elastic layer were proved to show their effect on the dynamic response of the double system. Its low response is searched.
\end{abstract}

Keywords: prestressed beam, string, dynamic excitation.

\section{Introduction}

Many years standing effort has been devoted to damp the dynamic effects of both the highway and railway vehicles when they cross a bridge. For that purpose, a lot of systems were developed, e.g. elastic supports of bridges, triangular falsework system with controlled damping, double systems with two beams or two strings connecting together with an elastic layer, etc. They are briefly described in [1] and cited in details in [2] and [3]. They are, especially, the papers of Kawanazoe et al. [4] and Oniszczuk [5], who firstly introduced the double beam and double string system, respectively. However, each of the systems mentioned above shows its technical or economic effectiveness only in some specific conditions. 
On the other hand, the prestressed bridges, world wide used for highway as well as railway bridges of small and medium spans, form naturally a double system with two elements: beam and pretensiled strings. That's the reason, why an idea arose - to bind both the elements with an elastic layer and dampers to diminish the dynamic response.

The aim of the present paper is to show the effect of the double system beam and string and put a question on the effectiveness of damping.

\section{Theory}

The Fig. 1 represents the theoretical model of a beam, pretensiled string and an elastic layer subjected to a row of axle forces. The axle forces $F_{n}, n=1,2,3, \ldots, N$, move with a constant speed $c$ from the left hand side to the right one. The simple supported beam and string provide the span $l$. The beam is subjected to an axial force $N_{1}$ (generally tension), while the string is tensed by a force $N_{2}$ (in practice, of course, $N_{1}=-N_{2}$ ). An elastic layer of Winkler type binds together both the carrying elements, its characteristic is $k$ $\left[\mathrm{N} / \mathrm{mm}^{2}\right]$ and its viscous damping $\omega_{d 1}$ or $\omega_{d 2}$, respectively.
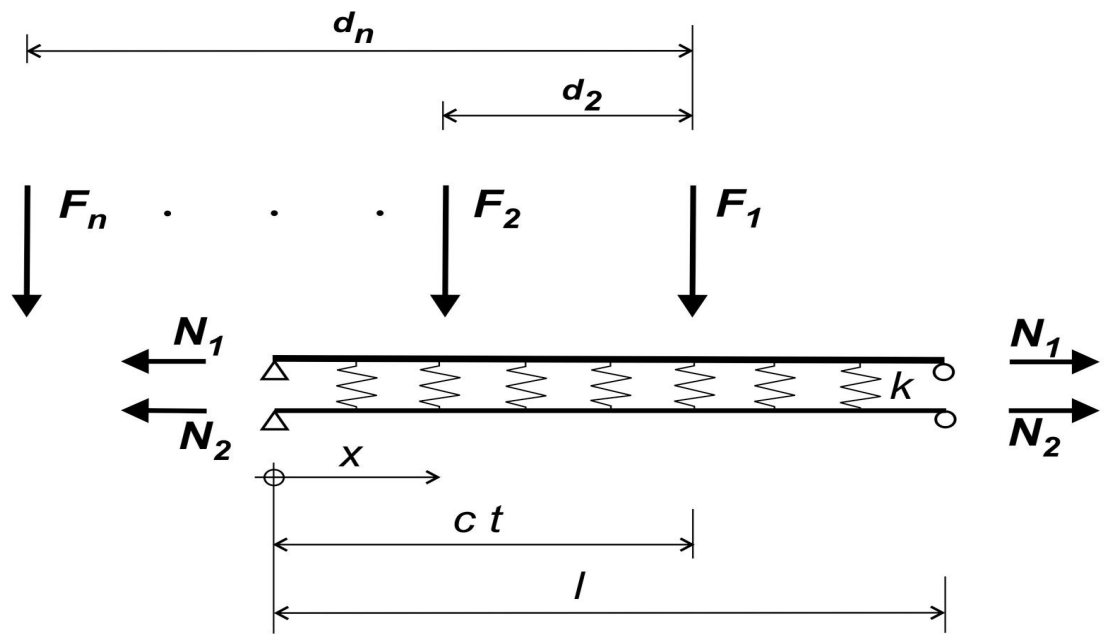

Figure 1: $\quad$ Theoretical model.

A system of partial differential equations describes the dynamic behaviour of the Bernoulli-Euler beam and string: 


$$
\begin{gathered}
E I \frac{\partial^{4} v_{1}(x, t)}{\partial x^{4}}-N_{1} \frac{\partial^{2} v_{1}(x, t)}{\partial x^{2}}+\mu_{1} \frac{\partial^{2} v_{1}(x, t)}{\partial t^{2}}+k\left[v_{1}(x, t)-v_{2}(x, t)\right]+ \\
+2 \mu_{1} \omega_{d 1}\left[\frac{\partial v_{1}(x, t)}{\partial t}-\frac{\partial v_{2}(x, t)}{\partial t}\right]=\sum_{n=1}^{N} F_{n} \varepsilon_{n}(t) \delta\left(x-x_{n}\right) \\
-N_{2} \frac{\partial^{2} v_{2}(x, t)}{\partial x^{2}}+\mu_{2} \frac{\partial^{2} v_{2}(x, t)}{\partial t^{2}}+k\left[v_{2}(x, t)-v_{1}(x, t)\right]+ \\
+2 \mu_{2} \omega_{d 2}\left[\frac{\partial v_{2}(x, t)}{\partial t}-\frac{\partial v_{1}(x, t)}{\partial t}\right]=0 .
\end{gathered}
$$

The following notations were applied:

$v_{i}(x, t), i=1,2$ - vertical deflection of the beam $(i=1)$ and the string $(i=2)$, respectively, at place $x$ and time $t$,

$E, I$ - modulus of elasticity and inertial cross-section moment, respectively, of the beam,

$\mu_{i}$ - mass of the beam $(i=1)$ and of the string $(i=2)$, respectively, per unit length,

$\delta(x)$ - Dirac delta function,

$\varepsilon_{n}(t)=h\left(t-t_{n}\right)-h\left(t-T_{n}\right)-$ the function describing the position of the $n$-th force with respect to the beam,

$h(t)=0$ for $t<0$ or $h(t)=1$ for $t \geq 0$, respectively - Heaviside unit function,

$d_{n}$ - distance of the $n$-th force from the first one, $d_{1}=0$,

$$
\begin{aligned}
& t_{n}=d_{n} / c ; \quad T_{n}=\left(l+d_{n}\right) / c, \\
& x_{n}=c t-d_{n} .
\end{aligned}
$$

The boundary and initial conditions, when the first force enters the beam, yield:

$$
\begin{gathered}
v_{1}(0, t)=v_{1}^{\prime \prime}(0, t)=v_{1}(l, t)=v_{1}^{\prime \prime}(l, t)=v_{2}(0, t)=v_{2}(l, t)=0, \\
v_{i}(x, 0)=\dot{v}_{i}(x, 0)=0, i=1,2,
\end{gathered}
$$

where the primes and dots denote the derivatives with respect to $x$ or $t$, respectively.

Several natural frequencies appear in the system (1) and (2) (without damping), which characterize the dynamic behaviour of individual elements:

The circular natural frequency of a simple beam without string and axial force yields: 


$$
\omega_{j}^{2}=\frac{j^{4} \pi^{4}}{l^{4}} \frac{E I}{\mu_{1}}, j=1,2,3, \ldots, f_{j}=\frac{\omega_{j}}{2 \pi},
$$

that one with the axial force $N_{1}$ (tension) but without the string:

$$
\omega_{1 j}^{2}=\frac{j^{4} \pi^{4}}{l^{4}} \frac{E I}{\mu_{1}}+\frac{j^{2} \pi^{2}}{l^{2}} \frac{N_{1}}{\mu_{1}},
$$

while this one of the string with an axial force $N_{2}$

$$
\omega_{2 j}^{2}=\frac{j^{2} \pi^{2}}{l^{2}} \frac{N_{2}}{\mu_{2}} \text {. }
$$

Further on, the natural frequencies of the elastic layer

$$
\begin{array}{ll}
\omega_{1 k}^{2}=k / \mu_{1}, & \omega_{2 k}^{2}=k / \mu_{2}, \\
\omega_{1 j k}^{2}=\omega_{1 j}^{2}+\omega_{1 k}^{2}, & \omega_{2 j k}^{2}=\omega_{2 j}^{2}+\omega_{2 k}^{2}
\end{array}
$$

and, finally, the circular natural frequency of the coupled system beam and string reads:

$$
\Omega_{1,2}^{2}=\frac{1}{2}\left(\omega_{1 j k}^{2}+\omega_{2 j k}^{2}\right) \mp\left[\frac{1}{4}\left(\omega_{1 j k}^{2}-\omega_{2 j k}^{2}\right)^{2}+\omega_{1 k}^{2} \omega_{2 k}^{2}\right]^{1 / 2} .
$$

The forced frequency of the moving force is:

$$
\omega=\frac{\pi c}{l} \text {. }
$$

\section{Solution}

For the solution of eqns (1) and (2), the Fourier integral transformation method is applied, [6], in the variable $x$

$$
\begin{aligned}
& V_{i}(j, t)=\int_{0}^{l} v_{i}(x, t) v_{j}(x) \mathrm{d} x, \\
& v_{i}(x, t)=\sum_{j=1}^{\infty} \frac{\mu_{i}}{V_{i j}} V_{i}(j, t) v_{j}(x)
\end{aligned}
$$

and the Laplace-Carson method in the variable $t$ :

$$
\begin{gathered}
V_{i}^{*}(j, p)=p \int_{0}^{\infty} V_{i}(j, t) \mathrm{e}^{-p t} \mathrm{~d} t, \\
V_{j}(j, t)=\frac{1}{2 \pi \mathrm{i}} \int_{a-\mathrm{i} \infty}^{a+\mathrm{i} \infty} \frac{1}{p} V^{*}{ }_{i}(j, p) \mathrm{e}^{\mathrm{ip}} \mathrm{d} p,
\end{gathered}
$$


where it is denoted

$$
\begin{gathered}
v_{j}(x)=\sin \frac{j \pi x}{l}, \\
V_{i j}=\int_{0}^{l} \mu_{i} v_{j}^{2}(x) \mathrm{d} x=\mu_{i} l / 2 .
\end{gathered}
$$

The transformed solution is received after the application of eqns (11) and (13):

$$
V_{i}^{*}(j, p)=\sum_{n=1}^{N} \frac{2 j \omega}{\mu_{1} l} \frac{\Delta_{i}}{\Delta}, i=1,2,
$$

where

$$
\begin{aligned}
\Delta_{1}= & \frac{j \omega F_{n}}{\mu_{1}} \frac{p}{p^{2}+j^{2} \omega^{2}} \mathrm{e}^{-p d_{n} / c}\left(1-\cos j \pi \mathrm{e}^{-p l / c}\right)\left(p^{2}+2 \omega_{d 2} p+\omega_{2 j k}^{2}\right), \\
\Delta_{2}= & \frac{j \omega F_{n}}{\mu_{1}} \frac{p}{p^{2}+j^{2} \omega^{2}} \mathrm{e}^{-p d_{n} / c}\left(1-\cos j \pi \mathrm{e}^{-p l / c}\right)\left(2 \omega_{d 2} p+\omega_{2 k}^{2}\right), \\
\Delta= & \left(p^{2}+2 \omega_{d 1} p+\omega_{1 j k}^{2}\right)\left(p^{2}+2 \omega_{d 2} p+\omega_{2 j k}^{2}\right)-\left(2 \omega_{d 1} p+\omega_{1 k}^{2}\right) . \\
& .\left(2 \omega_{d 2} p+\omega_{2 k}^{2}\right) .
\end{aligned}
$$

In the first stage, we do not assume the damping $\left(\omega_{d i}=0\right)$, then for the expressions

$$
\begin{aligned}
& F_{1}(p)=\frac{p\left(p^{2}+\omega_{2 j k}^{2}\right)}{\left(p^{2}+j^{2} \omega^{2}\right)\left(p^{2}+\Omega_{1}^{2}\right)\left(p^{2}+\Omega_{2}^{2}\right)}, \\
& F_{2}(p)=\frac{p \omega_{2 k}^{2}}{\left(p^{2}+j^{2} \omega^{2}\right)\left(p^{2}+\Omega_{1}^{2}\right)\left(p^{2}+\Omega_{2}^{2}\right)},
\end{aligned}
$$

the transformation relations (27.54) and (27.56) from [6] may be found

$$
\begin{gathered}
f_{1}(t)=\frac{1}{A}\left[\left(-\Omega_{2} B \sin \Omega_{2} t+\Omega_{1} C \sin \Omega_{1} t-j \omega D \sin j \omega t\right)+\right. \\
\left.+\frac{\omega_{2 j k}^{2}}{j \omega \Omega_{1} \Omega_{2}}\left(j \omega \Omega_{1} B \sin \Omega_{2} t-j \omega \Omega_{2} C \sin \Omega_{1} t+\Omega_{1} \Omega_{2} D \sin j \omega t\right)\right], \\
f_{2}(t)=\frac{\omega_{2 j k}^{2}}{j \omega \Omega_{1} \Omega_{2} A}\left(j \omega \Omega_{1} B \sin \Omega_{2} t-j \omega \Omega_{2} C \sin \Omega_{1} t+\Omega_{1} \Omega_{2} D \sin j \omega t\right),
\end{gathered}
$$

where

$$
A=B C D \text {, }
$$




$$
\begin{aligned}
& B=j^{2} \omega^{2}-\Omega_{1}^{2}, \\
& C=j^{2} \omega^{2}-\Omega_{2}^{2}, \\
& D=\Omega_{1}^{2}-\Omega_{2}^{2} .
\end{aligned}
$$

In this way, the resulting deflections of the beam and string appear with respect to (12) and (14):

$$
\begin{aligned}
v_{i}(x, t)= & \sum_{j=1}^{\infty} \sum_{n=1}^{N} \frac{2 j \omega}{\mu_{1} l} F_{n}\left[f_{i}\left(t-t_{n}\right) h\left(t-t_{n}\right)-\cos j \pi f_{i}\left(t-T_{n}\right) h\left(t-T_{n}\right)\right] . \\
& \cdot \sin \frac{j \pi x}{l}, i=1,2
\end{aligned}
$$

where the mutual relation (27.10) from [6] is used.

\section{Numerical results}

The deflection-time histories were calculated for several hundreds of cases in 3 series of different bridges. The eqn (29) was used for the undamped series, while for the damped cases, the inverse Fourier transformation and the ordinary differential equation with direct integration proceeded. The following case is demonstrated here as an example: series $\mathrm{C}$ with parameters:

$l=30 \mathrm{~m}, E=3.2 \times 10^{4} \mathrm{~N} / \mathrm{mm}^{2}, I=1.347 \times 10^{12} \mathrm{~mm}^{4}, \quad \mu_{l}=1.1 \times 10^{-2} \mathrm{Ns}^{2} / \mathrm{mm}^{2}, F=$ $=4.8 \times 10^{5} \mathrm{~N}, N_{2}=5.6 \times 10^{6} \mathrm{~N}, k=100 \mathrm{Ns}^{2} / \mathrm{mm}^{2}, \mu_{2}=0.002 \mathrm{Ns}^{2} / \mathrm{mm}^{2}, \omega_{\mathrm{d} 1,2}=0.1 \mathrm{~s}^{-1}$ at low velocity $5 \mathrm{~km} / \mathrm{h}$ and at speed $70 \mathrm{~km} / \mathrm{h}$.The responses of the beam mid- spans (in dimensionless form) are depicted in Figs 2 and 3. A row of 10 vehicles with axle loads $F_{1}=F_{3}=1.6 \times 10^{5} \mathrm{~N}, F_{2}=F_{4}=4.8 \times 10^{5} \mathrm{~N}$ in distances $d_{1}=0, d_{2}=3 \mathrm{~m}$, $d_{3}=12 \mathrm{~m}, d_{4}=15 \mathrm{~m}$ (valid for Czech standard highways) and with the gaps of 9 $\mathrm{m}$ between the vehicles were assumed.
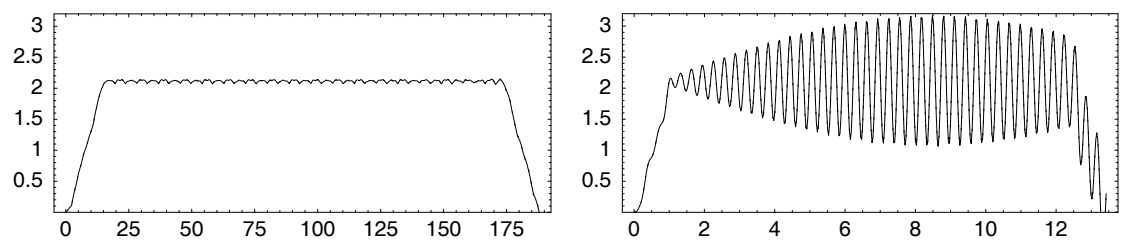

Figure 2: Deflection-time history at Figure 3: Deflection-time history at $5 \mathrm{~km} / \mathrm{h}$. $70 \mathrm{~km} / \mathrm{h}$.

The Figs 2 and 3 symbolize the great effect of the speed of moving forces. It proves again the conclusions in [6] and, moreover, the Fig. 3 shows the possibility of resonance vibration, [1]. The lines of $v_{1}(t)$ and $v_{2}(t)$ almost 
coincide in this case. It was derived in [2] and [3], that the eqns (1) and (2) depend on 6 dimensionless parameters. Then, it is difficult, particularly in practice, to develop the materials corresponding to the severe conditions for the parameters. Further on, the results in [2] and [3] show the ranges of parameters, which provide a low response of the beam subjected to a moving force.

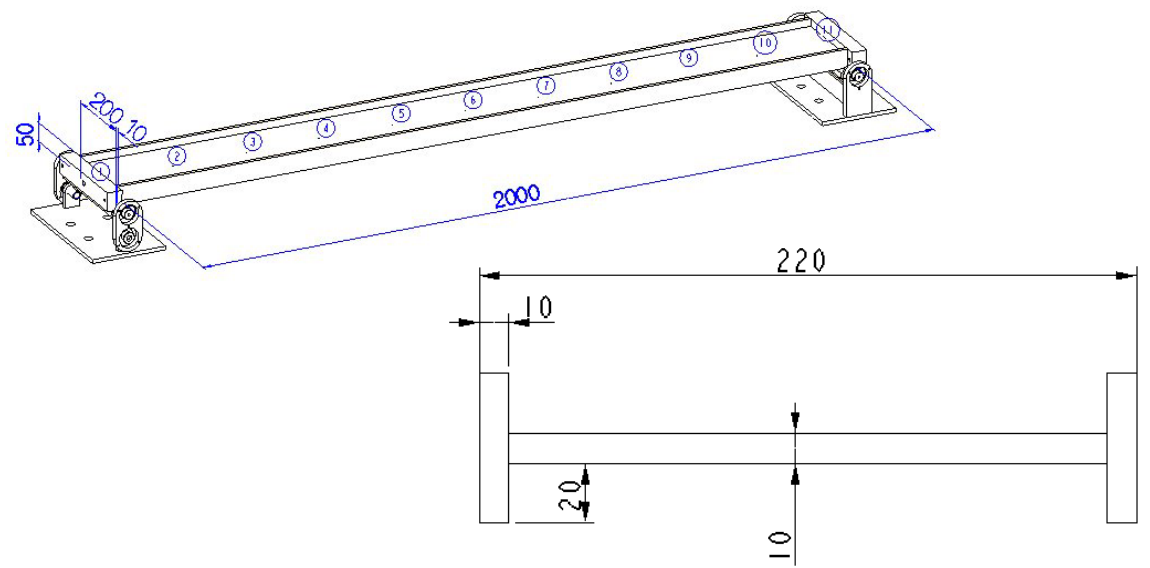

Figure 4: Laboratory model and cross-section of the beam.

\section{Experiments}

A simple laboratory model of the investigated problem was constructed. A plexiglass beam is prestressed by two steel wires, see Figs 4 and 5, whereas the electromagnetic exciter stimulates a harmonic force to the beam at several places. The response of the beam was also measured at several places. The special spring and damping elements, see Fig. 6, imititate the elastic layer. Several number of elements, distributed along the beam, were used for experiments.. The spring stiffness and damping characteristics were changed in three steps : maximum (max), mean (mean) and minimum (min) using the spring and liquid parts of the elements.

Figs 7 and 8 represent the beam acceleration $\ddot{v}$ (devided by the exciting force $F$ ) at $N_{2}=1000 \mathrm{~N}$ as a function of the three steps mentioned above. The figures show, how the arrangement (max, mean, min) diminishes the beam response. The designation $M P$ indicates the measuring point, where $M P 6$ is located at the midspan, while $M P$ 3(4) or $M P 9$ at one or three quarters of the span, respectively. The Fig. 7 shows the beam response to the exciter at the 1-st harmonics $5.16 \mathrm{~Hz}$, while the Fig. 8 that one at the 2-nd harmonics $22.18 \mathrm{~Hz}$. 


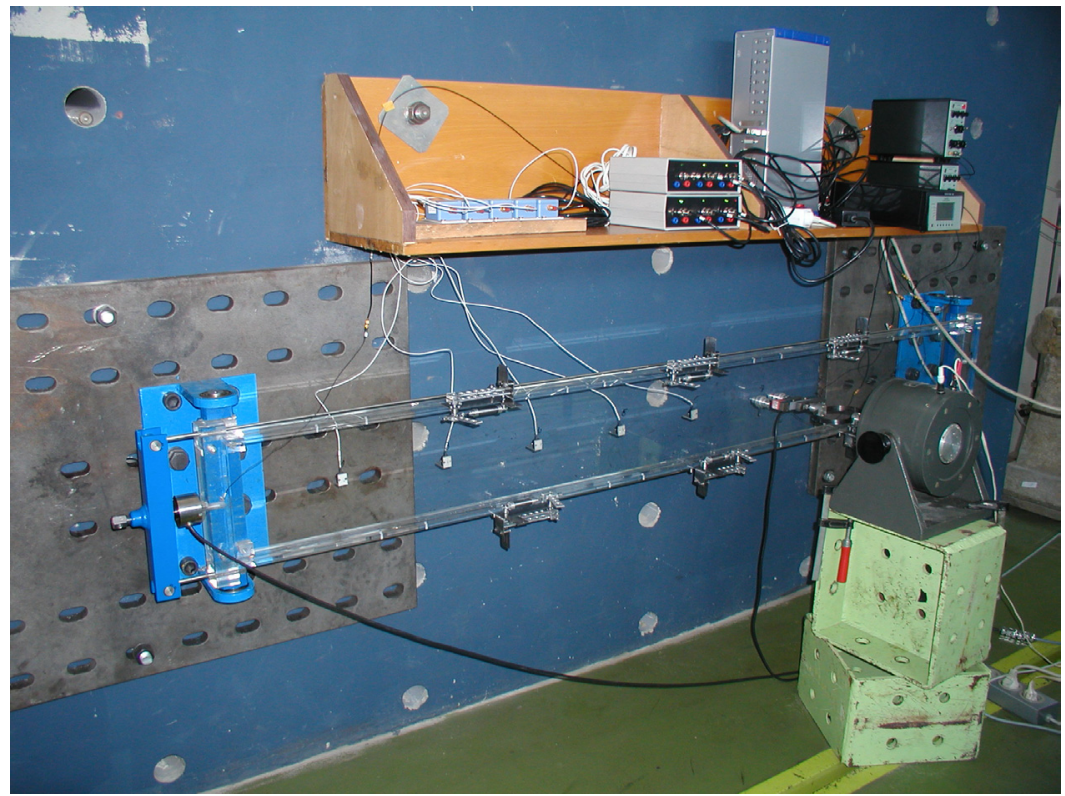

Figure 5: $\quad$ Model at laboratory tests.

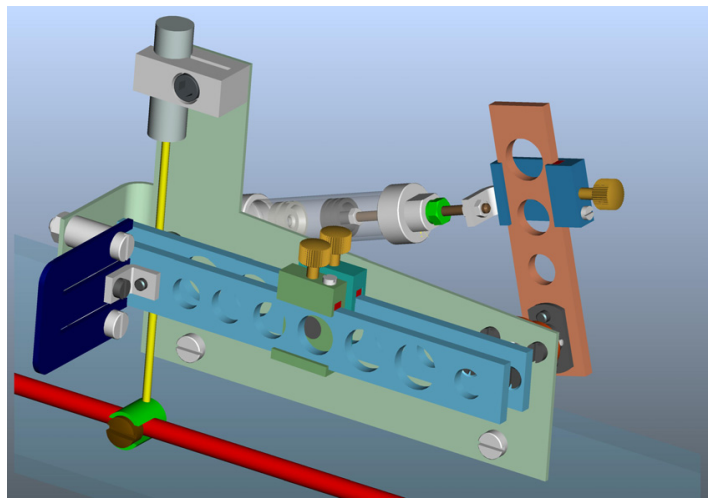

Figure 6: $\quad$ Spring and damping element.

\section{Conclusions}

The dynamic behaviour of the system beam and string bound together by an elastic layer is analysed. A set of partial differential equations describes the problem and is solved using the integral transformation methods. The equations are governed by several input parameters and, only in rear cases, the response of the beam with an elastic layer may be substantially lower than that one without the layer. 


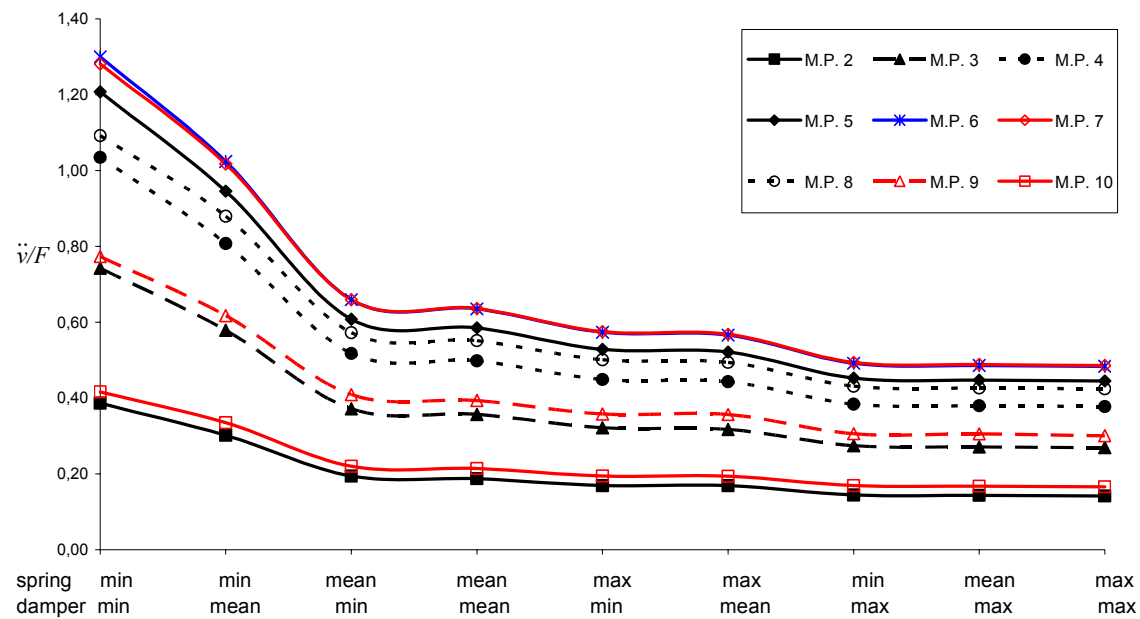

Figure 7: Damping of the beam with two spring and damping elements.

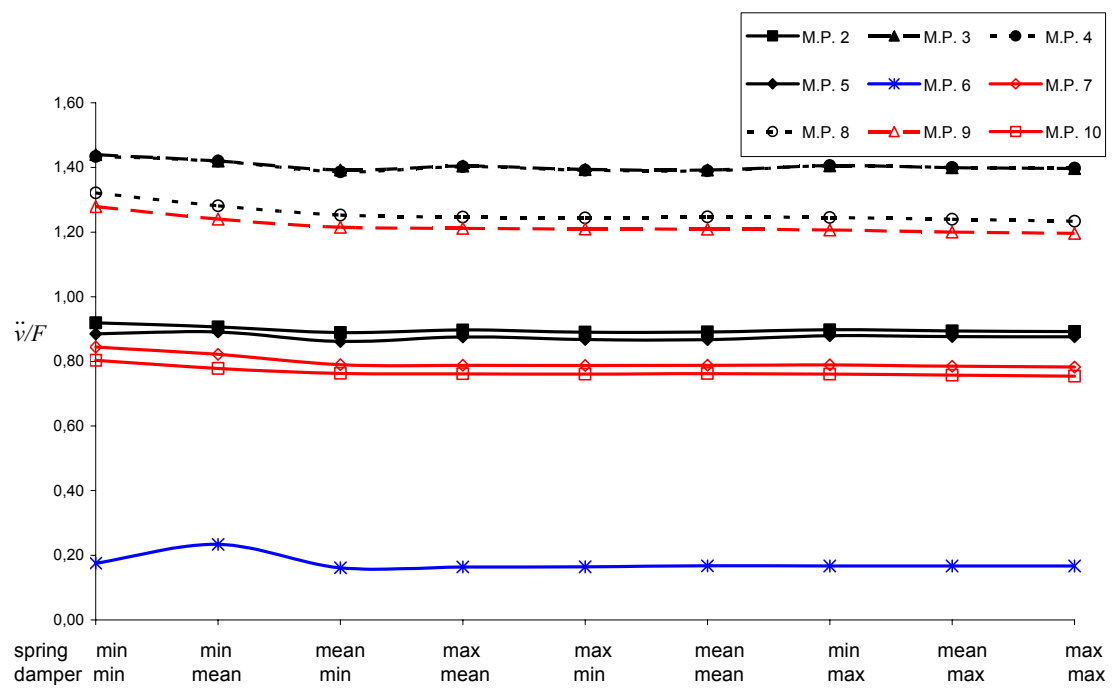

Figure 8: Damping of the beam with six spring and damping elements.

The laboratory experiments show the diminishing effect at several steps of stifffness and damping characteristics of the speciál elements on the response of the beam. The spring and damping elements affect, first of all, the first harmonics. A development of new damping layer materials would be necessary. Therefore, the outlook for the practical applications of the double system beam and strings seems to be rather pessimistic in near future. The main reason for the last conclusion is the low ratio of the string and beam masses. 


\section{Acknowledgements}

The supports of the grants GA CR 103/05/2066 and GA AS CR A200710505 as well as the institutional plan ITAM AV OZ 20710524 are gratefully ackowledged.

\section{References}

[1] Frýba, L., Dynamics of bridges under moving loads (past, present and future). In: D. Delgado, R. Calcada, J.M. Goicolea, F. Gabaldo (eds): Dynamics of High-Speed Railway Bridges. Porto, pp.25-44, 2005.

[2] Frýba, L. Fischer, C., Dynamics of prestressed beams coupled with a string. In: C.A. Brebbia, G.M. Carlomagno (eds): Computational Methods and Experimental Measurements XII. Southampton, Boston, WIT Press, pp. 445-454, 2005.

[3] Frýba, L., Fischer, C., Vibration of coupled system beam and string under a moving force. In: C. Soize, G.I. Schuëller (eds): Structural Dynamics EURODYN 2005, Paris. Millpress, Rotterdam, Netherlands, Vol. 2, pp. 1035-1037, 2005.

[4] Kawazoe, K., Kono, I., Aida, T., Aso, T., Eibisuda, K., Beam-type dynamics vibration absorber comprised of free-free beam. Journal of Engineering Mechanics, pp. 476-479, 1998.

[5] Oniszczuk, Z., Transverse vibration of elastically connected double-string system, Parts I, II. Journal of Sound and Vibration, 232, Vol. 2, pp. 355386, 2000.

[6] Frýba, L., Vibration of Solids and Structures Under Moving Loads. 3-rd ed., Academia, Prague, Thomas Telford, London, 1999. 